J. Appl. Numer. Optim. 1 (2019), No. 1, pp. 13-24

Available online at http://jano.biemdas.com

https://doi.org/10.23952/jano.1.2019.1.02

\title{
DC-GAP FUNCTION AND PROXIMAL METHODS FOR SOLVING NASH-COURNOT OLIGOPOLISTIC EQUILIBRIUM MODELS INVOLVING CONCAVE COST
}

\author{
LE DUNG MUU ${ }^{1, *}$, NGUYEN VAN QUY ${ }^{2}$ \\ ${ }^{1}$ Institute of Mathematics and Applied Sciences, Thang Long University, Hanoi, Vietnam \\ ${ }^{2}$ Academy of Finance, 8 Phan Huy Chu, Hoan Kiem, Hanoi, Vietnam
}

\begin{abstract}
We consider the Nash-Cournot oligopolistic equilibrium models involving separable concave cost functions. In contrast to the models with linear and convex cost functions, a local equilibrium point may not be a global one in the models. We propose two algorithms for finding global and local equilibrium points of the models having separable concave cost functions by using a DC decomposition of a gap function. The first algorithm uses the convex envelope of a separable concave cost function to approximate a concave cost model with affine cost ones. The latter is equivalent to strongly convex quadratic programs that can be solved efficiently. To obtain better approximate solutions, the first algorithm uses an adaptive rectangular bisection which is performed only in the space of concave variables. The second algorithm is an extension of the proximal method to the models.
\end{abstract}

Keywords. Adaptive rectangular bisection; Convex envelope; DC optimization formulation; Nash-Cournot oligopolistic model; Proximal point method.

2010 Mathematics Subject Classification. 47J20, 90C25.

\section{INTRODUCTION}

The Nash-Cournot oligopolistic market model is one of fundamental models in economics. In this model it is assumed that there are $N$-firms producing a common homogeneous commodity. Each firm $i$ has a strategy set $D_{i} \subset R_{+}$and a profit function $f_{i}$ defined on the strategy set $D:=D_{1} \times \ldots \times D_{N}$ of the model. Let $x_{i} \in D_{i}$ be a corresponding production level of firm $i$. Actually, each firm seeks to maximize its profit by choosing the corresponding production level under the presumption that the production of the other firms are parametric input. A commonly used approach to this model is based upon the famous Nash equilibrium concept.

We recall that a point (strategy) $x^{*}=\left(x_{1}^{*}, \ldots, x_{N}^{*}\right) \in D$ is said to be a Nash equilibrium point of this Nash-Cournot oligopolistic market model if

$$
f_{i}\left(x^{*}\right) \geq f_{i}\left(x^{*}\left[x_{i}\right]\right), \forall x_{i} \in D_{i}, \forall i
$$

where the vector $x^{*}\left[x_{i}\right]$ is obtained from $x^{*}$ by replacing $x_{i}^{*}$ by $x_{i}$.

\footnotetext{
*Corresponding author.

E-mail addresses: 1dmuu@math.ac.vn (L.D. Muu), quynv2002@yahoo.com (N.V. Quy).

Received January 29, 2019; Accepted March 10, 2019.
}

(C)2019 Journal of Applied and Numerical Optimization 
In the linear Nash-Cournot model the profit function of firm $i$ is given by

$$
f_{i}(x)=\left(\alpha_{i}-\beta \sum_{i=1}^{N} x_{i}\right) x_{i}-h_{i}\left(x_{i}\right)(i=1, \ldots, N) .
$$

where $\beta>0, \alpha_{i}>0, i=1, \ldots, N$ and, for every $i$, the cost function $h_{i}$ is affine that depends only on the quantity $x_{i}$ of firm $i$. In this linear case, it has been shown that (see, e.g., [6]) the model has a unique Nash equilibrium point which is the unique solution of a strongly convex quadratic program. In the case that $h_{i}$ is differentiable convex, the problem of finding a Nash equilibrium point can be formulated as a monotone variational inequality $[2,8]$ which can be solved by available methods for the monotone variational inequality.

In some practical applications, the cost for production of a unit commodity decreases as the quantity of the production gets larger. The cost function then is concave rather than convex. In this case as it is shown [9] that the problem can be formulated as a mixed variational inequality of the form

$$
\text { Find } x^{*} \in D:\left\langle F\left(x^{*}\right), x-x^{*}\right\rangle+\varphi(x)-\varphi\left(x^{*}\right) \geq 0, \quad \forall x \in D .
$$

In this problem $F$ is not monotone and $\varphi$ may not be convex. Therefore the existing methods for the monotone variational inequality cannot be applied. In [9], an algorithm is proposed for solving the model when some of the cost functions are piecewise linear concave. However the algorithm is efficient only when the number of the piecewise linear concave functions is relatively small. In [14], a proximal point method was described for finding a stationary point of the model.

A commonly used approach to nonconvex and generalized variational inequalities and generalized Nash equilibrium problems is the use of a gap function (see, e.g., $[3,4,7]$ and the references cited there).

In this paper we continue our work in [9] by using a DC optimization approach to the Nash-Cournot models where some of the cost functions are separable concave, the remaining costs are affine. Namely we use a suitable DC gap function for the problem and propose algorithms for solving the resulting DC programming problem thereby to obtain a global equilibrium point for the model. Thanks to the fact that the strategy set is a rectangle (box) and the cost functions are separable increasing, the gap function has particular features that can be employed to develop efficient algorithms for the model. We propose two algorithms: the first algorithm uses a branch-and-bound strategy while the second one can be considered as an extension of the proximal method in [18] to the model.

The rest part of the paper is organized as follows. In the next section we formulate the model as a DC optimization problem. The third section is devoted to description of the algorithms and their convergence results.

\section{A DC GAP FunCTION}

We recall that a function is said to be DC on a set if it can be decomposed into the difference of two convex functions on this set. The problem of optimizing a DC function over a convex set has been considered in many research papers, and some global as well as local optimization approaches have been developed for this problem (see, e.g., $[5,13,15,18]$ and the references therein). In this section, by using a gap function, we formulate the problem of finding an equilibrium point of a Nash-Cournot model involving concave cost functions as a DC optimization problem over the strategy set.

To be precise, we suppose that each profit function $f_{j}$ is defined by (1.1), where $h_{j}, j=1, \ldots, n$ is increasing concave while $h_{i}$ with $i>n$ is increasing affine. 
First, we define the bifunction $\phi$ by taking

$$
\phi(x, y):=\left\langle B_{1} x-a, y-x\right\rangle+y^{T} B y-x^{T} B x+h(y)-h(x)
$$

where

$$
B:=\left(\begin{array}{ccccc}
\beta & 0 & 0 & \ldots & 0 \\
0 & \beta & 0 & \ldots & 0 \\
\ldots & \ldots & \ldots & \ldots & \ldots \\
0 & 0 & 0 & 0 & \beta
\end{array}\right), B_{1}:=\left(\begin{array}{ccccc}
0 & \beta & \beta & \ldots & \beta \\
\beta & 0 & \beta & \ldots & \beta \\
\ldots & \ldots & \ldots & \ldots & \ldots \\
\beta & \beta & \beta & \ldots & 0
\end{array}\right)
$$

and

$$
h(x):=\sum_{i=1}^{N} h_{i}\left(x_{i}\right)
$$

Then the problem of finding an equilibrium point for the model can be formulated as a mixed variational inequality problem of the form (see, e.g., [10]):

$$
\left\{\begin{array}{l}
\text { find a point } x \in D \text { such that } \\
\Phi(x, y):=\left\langle B_{1} x-a, y-x\right\rangle+\varphi(y)-\varphi(x) \geq 0, \quad \forall y \in D
\end{array}\right.
$$

where

$$
\varphi(y):=y^{T} B y+h(y), \varphi(x):=x^{T} B x+h(x) .
$$

Clearly, $\varphi$ is a DC separable function.

Gap functions are commonly used to determine stoping rules in optimization and variational inequality and equilibrium problems as well as to formulate them into an optimization problem. Following this idea, we now define a gap function for the Nash-Cournot equilibrium models with separable concave cost functions. Namely, for the mixed variational inequality by taking, for each $x \in D$,

$$
g(x):=-\min \{\Phi(x, y): y \in D\} .
$$

Lemma 2.1. Suppose that cost function $h_{i}$ is continuous on $U_{i}$ for all $i=1,2, \ldots, N$. Then

(i) The gap function $g(x)$ is well defined, continuous and nonnegative on $D$.

(ii) A point $x^{*} \in D$ is equilibrium for the model if only if $g\left(x^{*}\right)=0$.

Proof. This lemma can be derived from Theorem 2.1 in [4]. Here we give a direct proof for the mixed variational inequality.

(i) Since $D$ is compact and for each $x \in D, \Phi(x,$.$) is continuous on D, \Phi(x,$.$) attains its minimum on$ $D$. Further, from property $\Phi(x, x)=0$, it follows that $g(x) \geq 0$ for every $x \in D$.

(ii) Suppose that $x^{*} \in D$ is an equilibrium point. Then

$$
\Phi\left(x^{*}, y\right) \geq 0, \quad \forall y \in U,
$$

which implies $g\left(x^{*}\right) \leq 0$ and $g\left(x^{*}\right)=0$. Conversely, if $g\left(x^{*}\right)=0$, then from the definition of $g\left(x^{*}\right)$ one has $\Phi\left(x^{*}, y\right) \geq 0$ for all $y \in D$, that means that $x^{*}$ is a equilibrium point of the model.

We rewrite the bifunction $\Phi$ as

$$
\Phi(x, y)=\left\langle B_{1} x-a, y-x\right\rangle+\beta \sum_{i=1}^{N} y_{i}^{2}+\sum_{i=1}^{N} h_{i}\left(y_{i}\right)-\beta \sum_{i=1}^{N} x_{i}^{2}-\sum_{i=1}^{N} h_{i}\left(x_{i}\right) .
$$


Gap function $g$ then can be rewritten as

$$
g(x)=-\min _{y \in U}\left\{\left\langle B_{1} x-\alpha, y-x\right\rangle+\beta \sum_{i=1}^{N} y_{i}^{2}+\sum_{i=1}^{N} h_{i}\left(y_{i}\right)\right\}+\beta \sum_{i=1}^{N} x_{i}^{2}+\sum_{i=1}^{N} h_{i}\left(x_{i}\right) .
$$

Since $D$ is the box of the form

$$
D:=\left\{x^{T}=\left(x_{1}, \ldots, x_{N}\right): 0 \leq l_{i} \leq x_{i} \leq u_{i}, i=1, \ldots, N\right\},
$$

we can further write $g(x)$ as

$$
\begin{aligned}
g(x)=- & \sum_{i=1}^{N} \min _{l_{i} \leq y_{i} \leq u_{i}}\left\{\left(B_{1} x-\alpha\right)_{i}\left(y_{i}-x_{i}\right)+\beta y_{i}^{2}+h_{i}\left(y_{i}\right)\right\} \\
& +\beta\left(\sum_{i=1}^{N} x_{i}\right)^{2}+\sum_{i=1}^{N} h_{i}\left(x_{i}\right) .
\end{aligned}
$$

A simple arrangement using (2.4) yields

$$
\begin{aligned}
g(x)=- & \sum_{i=1}^{N} \min _{l_{i} \leq y_{i} \leq u_{i}}\left\{\beta y_{i}^{2}+\left(\beta \sigma_{(-i)}(x)-\alpha\right) y_{i}+h_{i}\left(y_{i}\right)\right\} \\
& +\beta\left(\sum_{i=1}^{N} x_{i}\right)^{2}-\alpha^{T} x+\sum_{i=1}^{N} h_{i}\left(x_{i}\right)
\end{aligned}
$$

where $\sigma^{(-i)}(x):=\sum_{j \neq i}^{N} x_{j}$. Since the minimum of affine functions is concave, gap function $g$ is DC. For evaluating $g(x)$, for each $x \in D$, one needs to solve $N$-optimal problems each of them is one-variable

$$
\min _{l_{i} \leq y_{i} \leq u_{i}}\left\{\beta y_{i}^{2}+\left(\beta \sigma^{(-i)}(x)-\alpha\right) y_{i}+h_{i}\left(y_{i}\right)\right\} ; i=1,2, \ldots, N .
$$

When $h_{i}().(i=1,2, \ldots, p \leq N)$ are differentiable concave, (2.6) are one-dimensional smooth DC optimal problems.

\section{Description of the Algorithms}

In this section we describe a DC-gap function algorithm for approximating a global equilibrium point of the model. The algorithm is a branch-and-bound procedure, where the bounding operation uses the convex envelope of the "concave part" of the gap function, while the branching is an adaptive rectangular bisection involving only the concave variables.

\section{A branch-and-bound algorithm}

First we recall [5] that the convex envelope of a function $\varphi$ on a convex set $C$ is the convex function on $C$, denoted by $\operatorname{co}_{C} \varphi$ such that $\operatorname{co}_{C} \varphi(x) \leq \varphi(x)$ for every $x \in C$, and if $\xi$ is any convex function on $C$ satisfying $\xi(x) \leq \varphi(x)$ for every $x \in C$, then $\xi(x) \leq \cos _{C} \varphi(x)$ for every $x \in C$. It is well known [5] that the convex envelope of a concave function is affine. If $C=C_{1} \times \ldots \times C_{N}$ and $\varphi$ is separable, i.e., $\varphi\left(x_{1}, \ldots, x_{N}\right)=\sum_{j=1}^{N} \varphi_{j}\left(x_{j}\right)$, then $\operatorname{co} \varphi(x)=\sum_{j=1}^{N} \operatorname{co} \varphi_{j}\left(x_{j}\right)$, where $\operatorname{co} \varphi_{j}$ is the convex envelope of $\varphi_{j}$ over $C_{j}$. Clearly, since $h_{i}, i>n$ is affine, $h_{i} \equiv \operatorname{coh}_{i}$ on every convex set.

The algorithm is a branch-and-bound procedure for minimizing gap function $g$ using its DC decomposition obtained in the preceding section, thereby to obtain a global equilibrium point of the model. In this algorithm, the branching operation is a rectangular bisection that takes place only in the space of concave cost functions. 
To be precise, we suppose that $D:=D_{1} \times D_{2}$, where $D_{1}:=I_{1}^{0} \times \ldots \times I_{n}^{0}, D_{2}:=I_{n+1}^{0} \times \ldots \times I_{N}^{0}$ and that $h_{j}(j=1, \ldots, n)$ is concave on $I_{j}$ for every $j=1, \ldots, n$ and $h_{i}$ is affine on $I_{i}^{0}$ for every $i=n+1, \ldots, N$. Let

$$
I:=I_{1} \times \ldots \times I_{n}
$$

be a $n$-dimensional subbox of $D_{1}$, where $I_{j}:=\left[l_{j}, u_{j}\right]$ for every $j=1,2, \ldots, n$. An elementary computation shows that the convex envelope of the concave function $h_{j}$ over $I_{j}$ is

$$
\operatorname{co}_{I_{j}} h_{j}\left(x_{j}\right)=a^{I_{j}} x_{j}+b^{I_{j}},
$$

with

$$
\begin{aligned}
a^{I_{j}} & =\frac{h_{j}\left(u_{j}\right)-h_{j}\left(l_{j}\right)}{u_{j}-l_{j}}, \\
b^{I_{j}} & =\frac{u_{j} h_{j}\left(l_{j}\right)-l_{j} h_{j}\left(u_{j}\right)}{u_{j}-l_{j}}(j=1,2, \ldots, n)
\end{aligned}
$$

We use the DC decomposition $g(x)=g_{1}(x)-g_{2}(x)$ obtained by (2.5), where

$$
\begin{aligned}
g_{1}(x) & :=-\sum_{i=1}^{N} \min _{l_{i} \leq y_{i} \leq u_{i}}\left\{\beta y_{i}^{2}+\left(\beta \sigma_{(-i)}(x)-\alpha\right) y_{i}+h_{i}\left(y_{i}\right)\right\}+\beta\left(\sum_{i=1}^{N} x_{i}\right)^{2}, \\
g_{2}(x) & :=\alpha^{T} x-\sum_{i=1}^{N} h_{i}\left(x_{i}\right),
\end{aligned}
$$

For each $n$-dimensional subbox $I=I_{1} \times \ldots \times I_{n}$ of $D_{1}$, we define

$$
D_{I}:=\left\{x^{T}=\left(x_{1}, \ldots, x_{N}\right) \in D:\left(x_{1}, \ldots, x_{n}\right) \in I\right\}
$$

and

$$
c o_{I}\left(-g_{2}(x)\right):=-\alpha^{T} x+\sum_{j=1}^{n} c o_{I_{j}} h_{j}\left(x_{j}\right)+\sum_{j=n+1}^{N} h_{j}\left(x_{j}\right),
$$

where $c o_{I_{j}} h_{j}$ stands for the convex envelope of $h_{j}$ on $I_{j}$. The lower bound of $g$ over $D_{I}$ then can be defined by taking

$$
\beta\left(D_{I}\right):=\min _{x \in D_{I}}\left\{g_{1}(x)+c o_{I}\left(-g_{2}(x)\right)\right\} .
$$

In order to reduce the difference between function $h_{i}$ and its convex envelope, it suggests the use of rectangular bisections, which are suitable for separable global optimization problems [1, 5]. The following adaptive rectangular bisection will be used for the algorithms to be described below.

\section{An adaptive rectangular bisection (Rule 1).}

Let $I$ be a given $n$-dimensional subbox of $D_{1}$. Define

$$
j_{\text {max }}:=\operatorname{argmax}_{1 \leq j \leq n}\left\{h_{j}\left(x_{j}^{I}\right)-\operatorname{coh}_{j}\left(x_{j}^{I}\right)\right\} .
$$

Then we bisect $I$ into two boxes via the middle point of edge $I_{j_{\max }}$. We call this middle point the bisection point and $j_{\max }$ the bisection index.

For this bisection we have the following lemma whose proof can be found in [11].

Lemma 3.1. Let $\left\{I^{k}\right\}$ be an infinite sequence of boxes generated by the adaptive rectangular bisection Rule 1 such that $I^{k+1} \subset I^{k}$ for every $k$. Let $b^{k}$ be the bisection point and $j_{k}$ be the bisection index for $I^{k}$. Then $\lim _{k \rightarrow \infty}\left(h_{j_{k}}\left(b^{k}\right)-c_{I^{k}} h_{j_{k}}\left(b^{k}\right)\right)=0$. Consequently, $\left\{I_{j_{k}}\right\}$ tends to a singleton provided that $h_{j_{k}}$ is (concave) not affine on $I_{j_{k}}$. 
For each subbox $I$ having $n$-edges $I_{j}(j=1, \ldots, n)$, we define

$$
\rho\left(I_{j}\right):=\max _{t \in I_{j}}\left\{h_{j}(t)-\operatorname{coh}_{j}(t)\right\}
$$

and

$$
\rho(I):=\max \left\{\rho\left(I_{j}\right): j=1, \ldots, n\right\} .
$$

Motivated by Lemma 2.1, for an $\varepsilon \geq 0$, we call $x \in D$ an $\varepsilon$-equilibrium point if $0 \leq g(x) \leq \varepsilon$. The algorithm then can be described as follows.

Algorithm 3.1. (Branch-and bound)

Initial step. Choose a tolerance $\varepsilon \geq 0$ and set the initial box

$$
I^{0}:=I_{1}^{0} \times \ldots \times I_{n}^{0}=D_{1} .
$$

Solve convex program $\mathrm{P}\left(D_{I^{0}}\right)$ to obtain its optimal solution $x^{0}$ and a lower bound

$$
\beta_{0}:=\beta\left(D_{I^{0}}\right)=g_{1}\left(x^{0}\right)-\alpha^{T} x^{0}+\sum_{j=1}^{n} \operatorname{co}_{I_{j}^{0}} h_{j}\left(x_{j}^{0}\right)+\sum_{j=n+1}^{N} h_{j}\left(x_{j}^{0}\right)
$$

of $g$ on $D_{I^{0}}$. Let $\Gamma_{0}:=\emptyset$ if $\beta_{0}>0$. Otherwise, let

$$
\Gamma_{0}:=\left\{I^{0}\right\} ; \operatorname{vgap}_{0}:=g\left(x^{0}\right),
$$

$\rho_{0}:=\rho\left(I^{0}\right)$ and $\sigma_{0}:=n \rho_{0}$.

Iteration $k(k=0,1, \ldots)$. At each iteration $k$ we have a family $\Gamma_{k}$ of subboxes of $D_{1}$. To each $I \in \Gamma_{k}$ we associate a number $\beta\left(D_{I}\right)$ as the lower bound of $g$ over $D_{I}$ and the number $\rho(I)$. Moreover, we have the current largest lower bound $\beta_{k}$, the current best feasible point $x^{k}$ (the upper bound is always 0 ) and $\rho_{k}:=\max \left\{\rho(I): I \in \Gamma_{k}\right\}$.

Step 1 .

1a) If $\Gamma_{k}=\emptyset$, terminate: the model has nonequilibrium point.

1b) If $\operatorname{vgap}_{k} \leq \varepsilon$, terminate: $x^{k}$ is an $\varepsilon$-optimal solution.

Step 2. Choose $I^{k} \in \Gamma_{k}$ such that

$$
\rho_{k}:=\rho\left(I^{k}\right)=\max \left\{\rho(I): I \in \Gamma_{k}\right\}
$$

and let $\sigma_{k}:=n \rho_{k}$.

Step 3. Divide the subbox $I^{k}$ into two subboxes $I^{k^{+}}$and $I^{k^{-}}$by the bisection Rule 1 .

Step 4. Compute $\beta\left(D_{I^{+}}\right), \beta\left(D_{I^{k^{-}}}\right)$by solving Problems $\mathrm{P}\left(D_{I^{k^{+}}}\right)$and $\mathrm{P}\left(D_{I^{-}}\right)$. Let $x^{k^{+}}$and $x^{k^{-}}$be the obtained solutions of these problems respectively. Compute

$$
\operatorname{vgap}_{k+1}:=\min \left\{g\left(x^{k}\right), g\left(x^{k^{+}}\right), g\left(x^{k^{-}}\right)\right\} .
$$

Step 5. Update the the currently best feasible solution by taking $x^{k+1} \in\left\{x^{k}, x^{k^{+}}, x^{k^{-}}\right\}$such that

$$
g\left(x^{k+1}\right):=\min \left\{g\left(x^{k}\right), g\left(x^{k^{+}}\right), g\left(x^{k^{-}}\right)\right\} .
$$

Step 6. Set

$$
\Gamma_{k+1}:=\left\{I \in\left(\Gamma_{k} \backslash\left\{I^{k}\right\}\right) \cup\left\{I^{k+}, I^{k-}\right\}: \beta\left(D_{I}\right) \leq 0\right\}
$$

and update the currently best lower bound by taking

$$
\beta_{k+1}:=\min \left\{\beta(I): I \in \Gamma_{k+1}\right\}
$$


(we take $\beta_{k+1}=+\infty$ if $\Gamma_{k+1}=\emptyset$ ).

Compute $\rho\left(I^{k+}\right)$ and $\rho\left(I^{k-}\right)$ and take

$$
\rho_{k+1}:=\max \left\{\rho(I): I \in \Gamma_{k+1}\right\} ; \sigma_{k+1}:=n \rho_{k+1}
$$

(we take $\sigma_{k+1}:=0$ if $\Gamma_{k+1}=\emptyset$ ).

Let $k:=k+1$ and go to Step 1 .

Theorem 3.1. (Convergence Theorem). (i) If the algorithm terminates at iteration $k$, then $x^{k}$ is an $\varepsilon$ equilibrium point or else the model has nonequilibrium point (case 1b).

(ii) If the algorithm does not terminate, it generates an infinite sequence $\left\{x^{k}\right\}$ such that any its cluster point is an equilibrium point whenever the model has an equilibrium point. Furthermore $g\left(x^{k}\right) \searrow 0$ as $k \rightarrow \infty$.

Proof. The statement (i) is obvious from the stopping criteria.

(ii) For each iteration $k$ we have

$$
\Gamma_{k}:=\left\{I \subseteq D_{1}, \cup I=D_{1}\right\}
$$

and

$$
\Sigma_{k}:=\left\{I \times D_{2}, I \in \Gamma_{k}\right\} .
$$

Moreover, for each $x=\left(x^{1}, x^{2}\right) \in D=D_{1} \times D_{2}$, we define the function

$$
G_{k}(x)=g_{1}(x)-\alpha^{T} x+\operatorname{co} I(x), \text { if } x_{1} \in I \in \Gamma_{k},
$$

where

$$
\operatorname{co} h(x):=\sum_{j=1}^{n} \operatorname{co}_{I_{j}} h_{j}\left(x_{j}\right)+\sum_{j=n+1}^{N} h_{j}\left(x_{j}\right) .
$$

Obviously, by the definition then for each fixed $k$ and for all $x \in D$ we have

$$
G_{k}(x) \leq g(x)
$$

From Lemma 2.1, it follows that

$$
\beta_{k}=\min _{x \in D}\left\{G_{k}(x)\right\} \leq \min _{x \in D}\{g(x)\}=0
$$

and $\left\{\beta_{k}\right\}$ is nonincreasing. Then

$$
\lim _{k \rightarrow \infty} \beta_{k} \leq 0
$$

O the ther hand, we have

$$
0 \leq g(x)-G_{k}(x) \leq \sigma_{k}, \forall k, \forall x \in D
$$

which is equivalent to

$$
G_{k}(x) \geq g(x)-\sigma_{k}, \forall k, \forall x \in D .
$$

Taking the minimum of both sides, we obtain

$$
\min _{x \in D}\left\{G_{k}(x)\right\} \geq \min _{x \in D}\{g(x)\}-\sigma_{k},
$$

which implies

$$
\beta_{k} \geq-\sigma_{k}, \forall k
$$


Since $\sigma_{k} \rightarrow 0$ as $k \rightarrow \infty$, we have

$$
\lim _{k \rightarrow \infty} \beta_{k} \geq 0
$$

Combining (3.5) and (3.6) we obtain

$$
\lim _{k \rightarrow \infty} \beta_{k}=0
$$

Note that

$$
0 \leq g\left(x^{k}\right)-G_{k}\left(x^{k}\right) \leq \sigma_{k}, \forall k .
$$

Letting $k \rightarrow+\infty$ and recalling that $\left\{g\left(x^{k}\right)\right\}$ is the nonincreasing, we can write

$$
\lim _{k \rightarrow+\infty} g\left(x^{k}\right) \leq \lim _{k \rightarrow+\infty} \beta_{k}+\lim _{k \rightarrow+\infty} \sigma_{k}=0 .
$$

Then, using the fact $g(x) \geq 0, \forall x \in D$, we can deduce that

$$
\lim _{k \rightarrow+\infty} g\left(x^{k}\right)=0
$$

Now, suppose that $\bar{x}$ is a cluster point of $\left\{x^{k}\right\}$ and let $\left\{x^{k_{j}}\right\}$ is a subsequence of $\left\{x^{k}\right\}$ such that $x^{k_{j}} \rightarrow \bar{x}$ as $j \rightarrow+\infty$. By (3.7) and the continuity of $g$, we obtain

$$
\lim _{j \rightarrow+\infty} g\left(x^{k_{j}}\right)=g(\bar{x})=0 .
$$

Hence $\bar{x}$ is a global equilibrium point.

To illustrate the algorithm, let us consider the following example.

Example 3.1. We consider the model with the concave functions defined by

$$
h_{j}(x)=a_{j} x_{j}+\ln \left(1+\gamma_{j} x_{j}\right), j=1, \ldots, n,
$$

and the linear cost functions are defined by taking

$$
h_{j}\left(x_{j}\right)=\mu_{j} x_{j}, j=n+1, \ldots, N .
$$

The detail data of the model are taken as follows

$$
\begin{aligned}
& N=3 ; n=2 ; \\
& D=[0,100] \times[0,150] \times[0,50] ; \\
& D_{1}=[0,100] \times[0,150] ; D_{2}=[0,50] \\
& \alpha=(5,7,6) ; \beta=0.01 ; \\
& \gamma=(7,8) ; a=(2,3.5) ; \mu=(4) .
\end{aligned}
$$

An elementary computation shows that

$$
\begin{aligned}
g_{1}(x)= & -\min _{y_{1}}\left\{0.01 y_{1}^{2}+\left(0.01 x_{2}-3\right) y_{1}+\ln \left(1+7 y_{1}\right): 0 \leq y_{1} \leq 100\right\} \\
& -\min _{y_{2}}\left\{0.01 y_{2}^{2}+\left(0.01 x_{1}-3.5\right) y_{2}+\ln \left(1+8 y_{2}\right): 0 \leq y_{2} \leq 150\right\} \\
& +0.01\left(x_{1}+x_{2}+x_{3}\right)^{2}
\end{aligned}
$$

and

$$
g_{2}(x)=3 x_{1}+3.5 x_{2}+2 x_{3}-\ln \left(1+7 x_{1}\right)-\ln \left(1+8 x_{2}\right) .
$$

At each iteration, we use the subgradient projection algorithm for solving the nonsmooth convex program $P\left(D_{I}\right)$. The computations were executed on a PC Core 2 Duo $2 * 2.0 \mathrm{GHz}$, RAM $2 \mathrm{~GB}$. We take $\varepsilon=10^{-4}$.

- Initial step:

We solve the nonsmooth convex program $P\left(D_{I^{0}}\right)$ with 


$$
I^{0}=[0,100] \times[0,150] ; D_{I^{0}}=D=[0,100] \times[0,150] \times[0,50]
$$

and

$$
\operatorname{co}_{I^{0}}\left(-g_{2}(x)\right)=-2.9345 x_{1}-3.4527 x_{2}-2 x_{3}
$$

and obtain the following results

$$
\begin{cases}\beta_{0} & =-1.6704 \\ i t & =125 \\ x^{0} & =(80.3798,133.5634,0) \\ \text { vgap }_{0} & =g\left(x^{0}\right)=0.0572 \\ \rho_{0} & =1.6038 ; j_{\max }=1\end{cases}
$$

- Iteration $k=0$ :

Bisect the box $I^{0}=I^{0^{+}} \cup I^{0^{-}}$, where

$$
I^{0^{+}}=[0,50] \times[0,150] ; I^{0^{-}}=[50,100] \times[0,150] .
$$

For the subbox $I^{0^{+}}=[0,50] \times[0,150]$ we have

$$
\operatorname{co}_{I^{0^{+}}}\left(-g_{2}(x)\right)=-2.8828 x_{1}-3.4527 x_{2}-2 x_{3} .
$$

We solve the nonsmooth convex program $P\left(D_{I^{0^{+}}}\right)$and obtain

$$
\begin{cases}\beta_{I^{0^{+}}} & =4.8925 ; \\ i t & =141 ; \\ x^{I^{0^{+}}} & =(50,149.5708,8.3402) ; \\ \operatorname{vgap}_{I^{0^{+}}} & =g\left(x^{I^{0^{+}}}\right)=4.9099 \\ \rho_{I^{0^{+}}} & =1.3267 ; j_{\max }=1 .\end{cases}
$$

For the subbox $I^{0^{-}}=[50,100] \times[0,150]$, we have

$$
c o_{I^{0^{-}}} h(x)\left(-g_{2}(x)\right)=-2.9862 x_{1}-3.4527 x_{2}-2 x_{3}+5.1691 .
$$

For the nonsmooth convex program $P\left(D_{I^{0^{-}}}\right)$, we obtain

$$
\begin{cases}\beta_{I^{0^{-}}} & =-0.8050 \\ i t & =120 \\ x^{I^{0^{-}}} & =(86.0997,128.9940,0) \\ \operatorname{vgap}_{I^{0^{-}}} & =g\left(x^{I^{0^{-}}}\right)=0.0801 ; \\ \rho_{I^{0^{-}}} & =1.2225 ; j_{\max }=2 .\end{cases}
$$

Combining the results obtained the iteration $k=0$, we obtain

$$
\begin{cases}x^{1} & =(80.3798,133.5634,0) \\ \operatorname{vgap}_{1} & =0.0572 ; \\ \Gamma_{1} & =\{[50,100] \times[0,150]\} \\ \rho_{1} & =1.2225 ; j_{\max }=2 .\end{cases}
$$

- Iteration $k=1$ : 
Bisect the subbox $I^{1}=I^{1^{+}} \cup I^{1^{-}}$, where

$$
I^{1^{+}}=[50,100] \times[0,75], I^{1^{-}}=[50,100] \times[75,150] .
$$

For the subbox $I^{1^{+}}=[50,100]$, we have

$$
c o_{I^{1^{+}}}\left(-g_{2}(x)\right)=-2.9862 x_{1}-3.4147 x_{2}-2 x_{3}+5.1691 .
$$

Solving the nonsmooth convex program $P\left(D_{I^{1^{+}}}\right)$, we obtain

$$
\begin{cases}\beta_{I^{1^{+}}} & =15.0025 \\ i t & =30 \\ x^{I^{1^{+}}} & =(100,75,28.8450) \\ \operatorname{vgap}_{I^{0^{+}}} & =g\left(x^{I^{0^{+}}}\right)=15.0025 \\ \rho_{I^{0^{+}}} & =0.9542 ; j_{\max }=2 .\end{cases}
$$

For the subbox $I^{1^{-}}=[50,100] \times[75,150]$, we have

$$
c o_{I^{1^{-}}}\left(-g_{2}(x)\right)=-2.9862 x_{1}-3.4908 x_{2}-2 x_{3}+10.7422 .
$$

Solving the nonsmooth convex program $P\left(D_{I^{1^{-}}}\right)$, we obtain

$$
\begin{cases}\beta_{I^{1^{-}}} & =-0.0860 \\ i t & =124 ; \\ x^{I^{1^{-}}} & =(82.7369,133,2000,0) ; \\ \operatorname{vgap}_{I^{1^{-}}} & =g\left(x^{I^{1^{-}}}\right)=0.00007 .\end{cases}
$$

Combining the results obtained at the iteration $k=1$, we obtain

$$
\operatorname{vgap}_{2}=0.00007<\varepsilon=10^{-4} .
$$

So the algorithm terminates at iteration 2 and $x^{2}=(82.7369,133,2000,0)$ is an $\varepsilon$ - equilibrium point of the model.

Remark 3.1. The main subproblem in the proposed algorithm is the nonsmooth convex program $\mathrm{P}\left(D_{I}\right)$ for computing the lower bound $\beta\left(D_{I}\right)$. This convex mathematical program can be solved by some available algorithms, such as, the subgradient projection algorithm [12]. Efficiency of the algorithm depends on the efficiency of the algorithm used for the solving the nonsmooth convex subproblem $\mathrm{P}\left(D_{I}\right)$.

\section{A proximal point algorithm}

Motivated by the fact that a point $x^{*} \in D$ is an equilibrium point of the model if and only if the gap function $g\left(x^{*}\right)=0$, we say that a point $\bar{x} \in D$ is a local equilibrium point of the model if $\bar{x}$ is a local minimum of the gap function $g$ on $D$, that is, $g(\bar{x}) \leq g(x)$ for every $x \in B \cap D$, where $B$ is ball centered at $\bar{x}$. Because of the concavity of the cost function, in this equilibrium Nash-Cournot model, a local equilibrium point may not be a global one.

As we have seen, the Nash-Cournot model under consideration can be formulated as a mixed variational inequality of the form

$$
\text { Find } x \in D:\langle F(x), y-x\rangle+\varphi(y)-\varphi(x) \geq 0, \quad \forall y \in D,
$$


where

$$
F(x):=B_{1} x-a, \varphi(x)=\beta\|x\|^{2}+h(x),
$$

with $B_{1}$ being a matrix whose every diagonal entry is zero while all others are $\beta$, and $a$ being the $N$ dimensional vector whose every entry is $\alpha$.

It is clear that $x \in D$ is a solution of the above mixed variational inequality if and only if

$$
x \in \operatorname{argmin}\left\{\left\langle B_{1} x-a, y-x\right\rangle+\varphi(y)-\varphi(x): y \in D\right\} .
$$

Motivated by this fact, using the optimality condition for problem $P(x)$, we call $x \in D$ a stationary point of the above mixed variational inequality if

$$
0 \in c\left[F(x)+\nabla \varphi(x)+N_{D}(x)\right]
$$

where $c>0$ and $N_{D}(x)$ is the normal cone of $D$ at $x$. Remember that $\varphi(x)=g(x)-h(x)$ with $g$ and $h$ being convex functions. Let

$$
g_{1}(x):=g(x)+\delta_{D}(x),
$$

where $\delta_{D}($.$) is the indicator function for the convex set D$. Since $N_{D}(x)=\partial \delta_{D}(x)$, we see that $x$ is a stationary point if and only if

$$
0 \in c\left(\partial g_{1}(x)+F(x)-\nabla h(x)\right)
$$

where $c>0$. Since $g$ is lower semicontinuous convex function and $D$ is closed convex set, $\partial g_{1}$ is maximal monotone and

$$
\left(I+c \partial g_{1}\right)^{-1}
$$

is singleton, defined everywhere [16]. Note that $h$ is differentiable, by a simple arrangement (see also [17]), we can see that $x$ is a stationary point of the above mixed variational inequality if and only if

$$
x=\left(I+c \partial g_{1}\right)^{-1}(x-c F(x)+c \nabla h(x)) .
$$

Starting from a point $x^{0} \in D$, the proximal point algorithm for finding a point satisfying the fixed point problem (3.8) takes the form

$$
x^{k+1}=\left(I+c \partial g_{1}\right)^{-1}\left(x^{k}-c F\left(x^{k}\right)+c \nabla h\left(x^{k}\right)\right) .
$$

Let

$$
y^{k}:=x^{k}-c F\left(x^{k}\right)+\nabla h\left(x^{k}\right) .
$$

Then the problem of finding $x^{k+1}$ amounts to solving the following strongly convex program

$$
\min \left\{g(x)+\frac{1}{2 c}\left\|x-y^{k}\right\|^{2}: x \in D\right\} .
$$

Finally, we remark that if $F \equiv 0$, that is, $\beta=a=0$, then this proximal algorithm is reduced to the one studied in [18]. The convergence of the algorithm in this case can be found in [18]. It is of interest to continue to study the convergence of the general case. 


\section{REFERENCES}

[1] F.A. Al-Khayyal, Jointly constrained bilinear programs and related problems: An overview. Comput. Math. Appl. 19 (1990), 53-62.

[2] F. Facchinei, J.S. Pang, Finite-Dimensional Variational Inequalities and Complementarity Problems, Springer, Berlin, 2002.

[3] M. Fukushima, Equivalent differentiable optimization problems and descent methods for asymmetric variational inequality problems, Math. Program. 53 (1992), 99-110.

[4] M. Fukushima, J.S. Pang, Quasi-variational inequality, generalized Nash equilibria, and multi-leader-folower games, Comput. Management Sci. 2 (2005), 21-26.

[5] R. Horst, H. Tuy, Global Optimization (Deterministic Approach), Springer, Berlin, 1990.

[6] I. Konnov, Combined Relaxation Methods for Variational Inequalities, Springer, Berlin, 2001.

[7] K. Kubota, M. Fukushima, Gap function approach to the generalized Nash equilibrium problem, J. Optim. Theory Appl. 144 (2010), 511-531.

[8] H.F. Murphy, H.D. Sherali, A.L. Soyster, A mathematical programming approach for determining oligopolistic market equilibrium, Math. Program. 24 (1982) 92-106.

[9] L.D. Muu, V.H. Nguyen, N.V. Quy, On Nash-Cournot oligopolistic market models with concave cost functions, J. Global Optim. 41 (2007), 351-364.

[10] L.D. Muu, T.D. Quoc, One step from DC. optimization to DC. mixed variational inequalities, Optimization 59 (2010), 63-76.

[11] A. Nagurney, Network Economics: A Variational Inequality Approach, Kluwer Academic Publishers, Dordrecht, 1993.

[12] Y. Nesterov, Introductory Lectures on Convex Programming, Volume I: Basic Course CORE, 1998

[13] D.T. Pham, H.A. Le Thi, Convex Analysis approach to DC prohramming: Theory, algorithms and applications, Acta Math. Vietnamica 22 (1997), 289-355.

[14] T.D. Quoc, L.D. Muu, A spritting proximal point method for Nash-Cournot equilibrium models involving nonconvex cost functions, J. Nonlinear Convex Anal. 12 (2011), 519-534

[15] N.V. Quy, A vector optimization approarch to Cournot oligopolistic market Models, Int. J. Optim. Theory, Methods Appl. 1 (2009), 341-360.

[16] R.T. Rockafellar, Convex Analysis, Princeton Landmarks in Mathematics, Princeton University Press, Princeton, (1997)

[17] R.T. Rockafellar, Monotone operator and the proximal point algorithms, SIAM. J. Control Optim. 14 (1976), 877-898.

[18] W. Sun, R.J.B. Sampaio, M.A.B. Condido, Proximal point algorithm for minimization of DC function, J. Comput. Math. 21 (2003), 451-462. 